\title{
Coherent X-ray Imaging: Bridging the Gap between Atomic and Micro-scale Investigations
}

\author{
Marco Stampanoni"ab, Andreas Menzel ${ }^{a}$, Ben Watts ${ }^{a}$, Kevin S. Mader ${ }^{\text {ab }}$, and Oliver Bunk
}

\begin{abstract}
We present a review of state-of-the art X-ray imaging techniques based on partially coherent synchrotron radiation. Full-field X-ray tomography, $X$-ray ptychography, scanning small-angle X-ray scattering, and scanning transmission $X$-ray microscopy are imaging techniques that gather structural information at spatial resolution ranging from several microns to a few tens of nanometers in both real- and reciprocal space. These methods exploit contrast mechanisms based on absorption, phase, and spectroscopic signals. We provide examples of how these techniques can be applied to address scientific questions ranging from imaging of biological samples, to foam rheology, and cement composition.
\end{abstract}

Keywords: Coherent X-ray imaging · Ptychography · Scanning transmission microscopy · Small-angle X-ray scattering $\cdot$ Tomography

\section{Introduction}

Synchrotron X-ray facilities are well recognized as powerful tools for determining crystal structures with high precision. Although crystallography is a major activity at synchrotron facilities, there are many other techniques that take advantage of the unique properties of synchrotron light. The short wavelength and significant penetration depth of X-rays, coupled with the brilliance and coherence of synchrotron sources, provide an advantageous platform for a wide range of imaging techniques providing resolutions from the nanometer to the micrometer length scales. Thanks to a wide variety of contrast mechanisms, which can be tailored to suit the specific scientific questions at hand, synchrotronbased X-ray imaging techniques are making valuable scientific contributions across many disciplines.

\footnotetext{
${ }^{\star}$ Correspondence: Prof. Dr. M. Stampanoniab Tel.: +4156 3104724

E-mail: stampanoni@biomed.ee.ethz.ch

aPaul Scherrer Institute

$\mathrm{CH}-5232$ Villigen-PSI

bInstitute for Biomedical Engineering

ETH and University of Zurich

Gloriastrasse 35, CH-8092 Zürich
}

In this article we will discuss some of the techniques that are utilizing synchrotron radiation to provide unique imagery: X-ray tomography, X-ray ptychography, scanning small-angle X-ray scattering (scanning SAXS), and scanning transmission X-ray microscopy (STXM). The set of techniques we discuss represent a variety of physical approaches to gather structural information, covering both real- and reciprocal-space, full-field and scanning, and with contrast mechanisms utilizing absorption, phase, and spectroscopic signals. While far from being an exhaustive list of what is currently available, the highlighted techniques provide a broad overview of $\mathrm{X}$-ray imaging and how they can aid, for example, the life- and materials scientist.

\section{Full-field X-ray Microscopy and Tomography}

Tomographic X-ray microscopy is a powerful, non-destructive technique for the three-dimensional (3D) investigation of the internal structure of a variety of optically opaque samples. Introduced in 1973 by Hounsfield and mostly applied in the medical field initially, it is now a well established approach in many fields such as life, materials, food, pharmaceutical, environmental, and earth sciences to cite a few.

In the most common case of full-field, absorption-based tomographic microscopy, radiographic projections are acquired. The signals correspond to the line integral of the attenuation coefficient along the beam path; they are dominated by the photoelectrical effect and are strongly dependent on the energy $\left(1 / \mathrm{E}^{3}\right)$ of the probing ra- diation and on the atomic numbers $\left(Z^{3}\right)$ of the elements in the sample. Phase contrast $\mathrm{X}$-ray microscopy, on the other hand, is sensitive to refraction and shows a weaker dependence on energy (1/E). Radiographic projections only provide two-dimensional (2D) cumulative information on the structure along the beam path. However, 3D internal structural details can be ascertained by taking radiographic projections of the sample under different angles (usually equally distributed between 0 and $180^{\circ}$ ) and combining them with dedicated algorithms for tomographic reconstructions based, for instance, on Fourier analysis or iterative methods.

In addition to the simple visualization of internal 3D details, extraction of morphological and quantitative statistical information is now possible thanks to everincreasing computational power. Number, size, shape, orientation, spatial distribution, connectivity, and packing of features of interest within the sample are just a few of the possible quantitative measures that can be extracted from tomographic datasets. We present here two examples of typical quantitative analyses. Examples showing the static and dynamic quantitative capabilities are taken from bone mechanics and foam rheology, respectively. The lefthand panel of Fig. 1 depicts a cortical bone region located inside a mouse femur. The image has been segmented from absorption information and labeled to identify the porous structures inside the bone matrix (not shown). These porous structures are labeled as canals (larger structures) and osteocyte lacunae (small cellular structures) ${ }^{[1]}$ whose shapes can be analyzed in order to characterize the microstructure of the 
bone. Furthermore, relationships between these structures can be examined by creating surface distance maps (Fig. 1, left panel) showing the distance of each point inside the bone to the nearest canal surface. Average osteocyte and canals distances can then be calculated, and insights into the nutritional and communication pathways can be gained by looking at correlations between shape and distance.

The right-hand panel of Fig. 1 illustrates a snapshot of liquid foam moving through a flow chamber. The only visible material when imaging foams with X-rays is the liquid phase that separates adjacent bubbles, called plateau borders. The bubble position and shape must be inferred from the distance map of the borders using, for instance, a custom-developed algorithm. ${ }^{[2]}$ The bubbles can then be tracked using a nearest-neighbor algorithm, resulting in dynamic information about the rheology of the sample. This procedure provides full $3 \mathrm{D}$ information on the movements of the bubbles, their deformations, and topological rearrangements. This information is crucial to understanding the physics and ultimately the mechanical properties of such a system. Very often, such detailed volumetric information on the real specimen structure provides very realistic input data to simulation packages and enables more reliable predictions of system properties.

Thanks to the development of novel detectors during the last decades, X-ray imaging at high-brilliance third-generation synchrotron facilities has experienced a true revolution, enabling tomographic investigation at the sub-micrometer scale to be carried out in a fraction of a second. This allows the investigation of evolving systems, gives insight into the dynamics of the sample and, as a consequence, shifts the analysis from the classical, static anatomic contents to the more intriguing functional, 'physiological' aspects. This additional information can be collected using monochromatic radiation as a probe, eliminating beam hardening artifacts, which normally affect data acquired at polychromatic sources such as most laboratorybased setups. Quantitative measurements of material properties are thus possible and the identification of different phases more reliable. Further, the almost parallel beam geometry typical for tomographic microscopy endstations at synchrotron sources allows the accurate reconstruction of tomographic volumes. The resulting images are of astonishing quality and devoid of cone beam artifacts.

The most striking property of synchrotron beams is their partial coherence. This feature allows the generation of interference signals and, as a consequence, provides access to phase information in the investigated sample. The X-ray imaging community has been very much stimulated by this option, as it had been continually challenged by the question of how to increase the contrast in X-ray images without increasing the dose delivered to a specimen. From the Kramers-Kronig relations, it appears evident that the phase shift induced by a sample would yield a stronger contrast than that produced by absorption, theoretically by several orders of magnitude. An example of how the phase signal can enhance soft-tissue contrast within a sample is illustrated in Fig. 2. The picture shows a tomographic scan of a rat-brain (fixed in paraformaldehyde and embedded in paraffin) measured at the TOMCAT beamline of the Swiss Light Source using grating interferometry, a phase contrast technique de- veloped at the Paul Scherrer Institute over the last decade. ${ }^{[3-7]}$ The anatomical details in the phase image on the left are much more clearly delineated compared to those in the absorption image on the right, which are barely visible. It is important to note here that both images were obtained simultaneously, with the same dose delivered to different parts of the sample. Recording $\mathrm{X}$-ray phase contrast signals has proven to be quite difficult, unless the sample can be illuminated in a sufficiently coherent manner. Such illumination is an inherent advantage of third-generation synchrotron facilities over conventional X-ray sources. Recently, it has been shown, however, that grating-based X-ray interferometry can efficiently yield phase contrast images even with conventional X-ray sources ${ }^{[4]}$ as shown in Fig. 3. ${ }^{[8]}$ Panel (a) shows the classical, absorption-based image of a human hand, taken with a conventional X-ray source and a modern X-ray detector. This image contains the same physical information as the first radiograph obtained by Röntgen back in 1895 , namely the $2 \mathrm{D}$ distribution of the energy and material dependent line integrals of the attenuation coefficient. Panels b) and c) show the differential phase and dark-field signal respectively. In medical imaging, both these signals have so far been considered as a source of image quality deterioration and have therefore not been utilized. However, phase contrast and scattering-based X-ray imaging can potentially revolutionize the radiological approach to medical imaging due to their intrinsic capability of detecting subtle differences in the electron density of a material and of measuring the effective integrated local small-angle scattering power generated by the microscopic density fluctuations in the specimen.

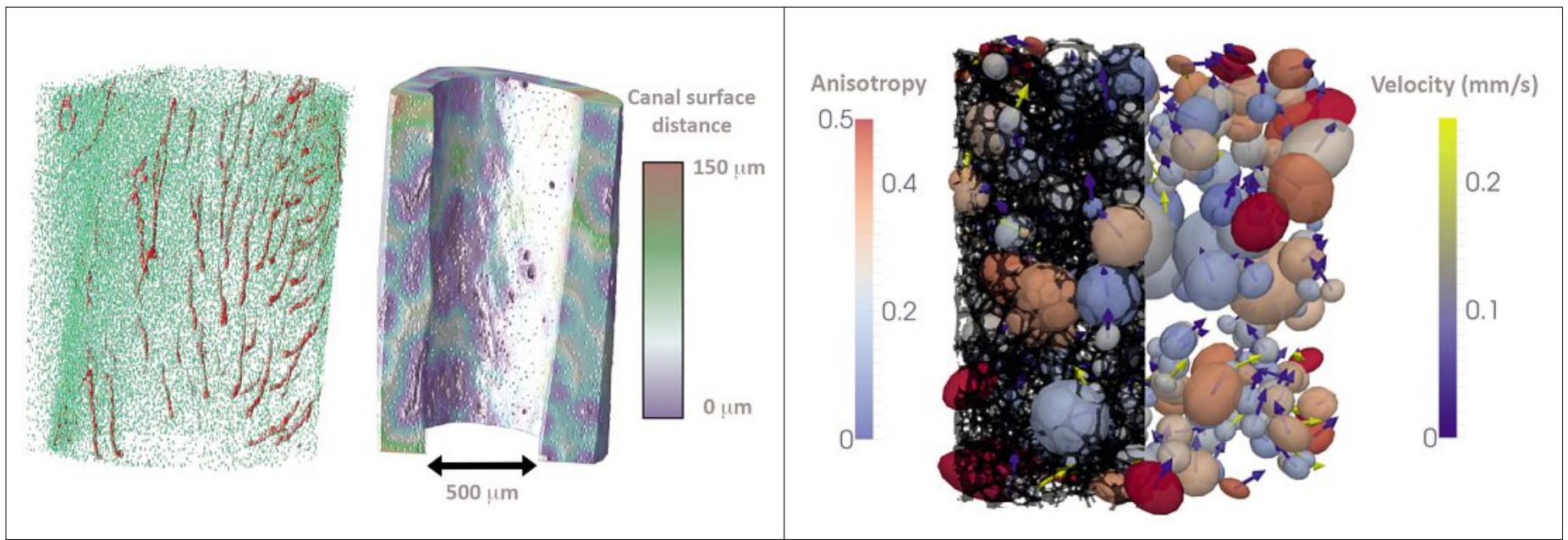

Fig. 1. Left panel: $3 \mathrm{D}$ rendering of cortical bone measured in the mid-diaphysis of a mouse femur. Left: canals colored in red and the osteocyte lacunae colored in turquoise. Right: distance map from the canal surface, where the distance for every point in the bone from the nearest canal surface is shown using a color between blue and red. Right panel: 3D rendering from a rheological experiment imaging liquid foam. Plateau borders (representing the visible liquid phase) are colored in black in the left half of the image. The air bubbles are shown with ellipsoids colored between blue and red to represent the degree of anisotropy of the bubble. The arrows indicate the direction of movement for each bubble. Their length is proportional to the bubble velocity in mm/s. (Courtesy of R. Mokso, C. Raufaste, B. Dollet, and S. Santucci, see ref. [1]) 


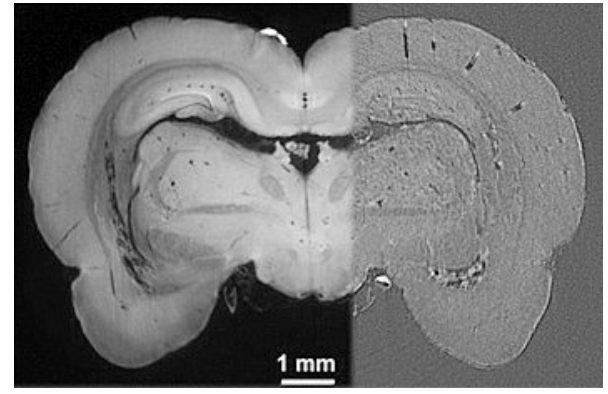

Fig. 2. Left side: phase-contrast tomographic reconstruction of a mouse brain measured with the grating interferometer installed at the TOMCAT beamline of the SLS, operated at 25 $\mathrm{keV}$ (isotropic spatial resolution of 15 microns, density sensitivity of $\left.1 \mathrm{mg} / \mathrm{cm}^{3}\right)$. Right side: absorption-based tomographic reconstruction of the same sample. See ref. [6].

\section{Ptychography}

In full-field imaging mode, the ability to resolve minute details of an object stems from either a magnified projection of the object onto an X-ray detector or from a magnifying lens. In the former approach image resolution is limited by the pixel size of the detector and by the size of the $\mathrm{X}$-ray source, which is often not the source itself but a demagnified image of it; in the latter case, resolution and image quality depend on the X-ray optical elements. However, despite impressive progress in manufacturing X-ray optics, ${ }^{[9,10]}$ the combination of high numerical aperture, which is a requirement for achieving high resolution, with high efficiency and low aberration remains a true challenge.

Mathematical routines that directly analyze the light scattered by the sample are an alternative to image-forming optics. ${ }^{[11]}$ Such lensless techniques are often subsumed under the term coherent diffractive imaging (CDI). Even at high-brilliance synchrotron sources, the necessary coherence filtering, i.e. monochromatizing the $\mathrm{X}$-rays and ensuring a well-defined direction of propagation, for instance by having a small source far away, ${ }^{[12]}$ typically reduces the usable flux by orders of magnitude. Given such careful preparation of the illuminating X-rays, CDI is widely believed to allow image resolution and sensitivity limited only by the radiation tolerance of the sample. Single-shot 'diffract before destroy' experiments at ultra-fast freeelectron laser (FEL) sources may bypass even this limitation. ${ }^{[13]}$

Typically, a small sample is illuminated with a plane wave, and its far-field scattering pattern is finely sampled. While the scattering process is well understood and characterized, inverting measurable intensity distributions to obtain an image of the sample, often referred to as image retriev-
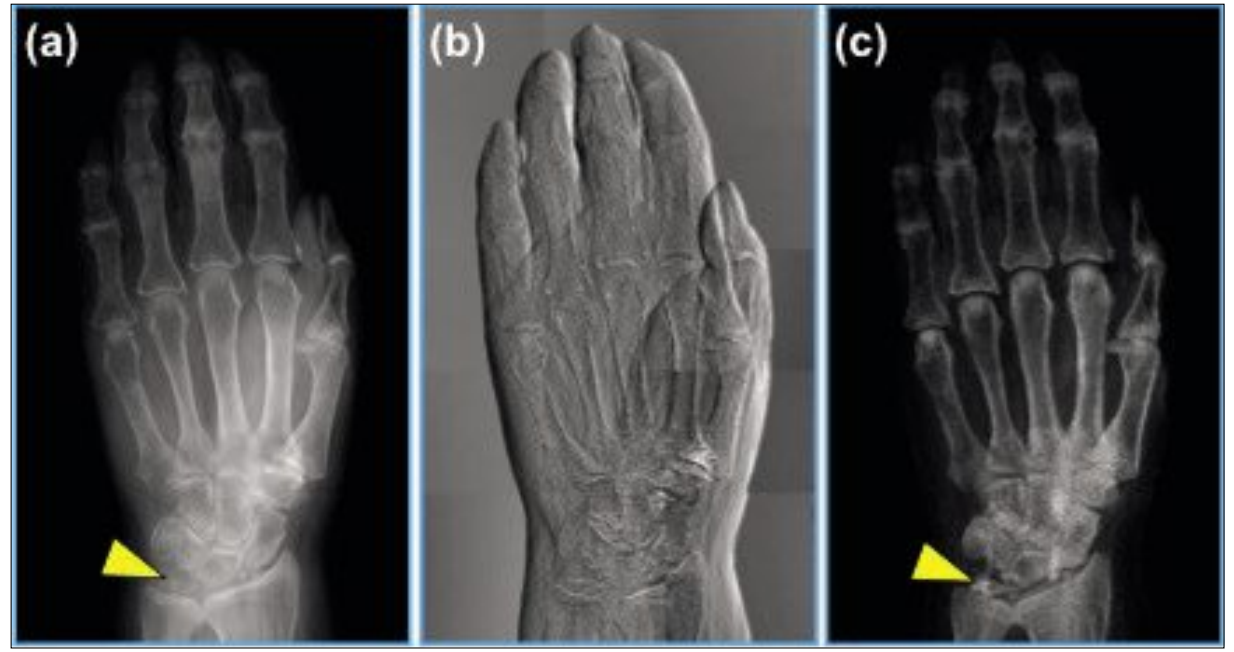

Fig. 3. Absorption (a), differential phase (b), and dark-field (c) signal from a human hand. Panel (b) clearly shows the soft tissue signal: muscles, tendons, and nails are visible. Panel (c) depicts the small-angle scattering signal. The arrowheads indicate a region of soft-tissue calcification in the triangular fibrocartilage. This region is not visible in the conventional absorption image (a). Data were obtained on a conventional X-ray source with a Talbot-Lau interferometer operated at 28 keV. See ref. [8]. Reproduced with permission from Taylor and Francis Book, Inc., 2012.

al, is a mathematically ill-posed problem, and special care has to be taken in order to ensure a unique solution. Image retrieval usually comprises many cycles of iterative processes that alternate in enforcing $a$ priori knowledge on the sample, such as its finite size, and consistency with measured data. Such 'standard' CDI requires the samples to be isolated and has a low tolerance to perturbations by signals due to other scatterers, e.g. from ice or the sample holder. In fact, extraneous scattering is the major impediment to the application of the technique.

Holography[14,15] and a technique called ptychography are significantly more robust. The two techniques were originally developed in the $1940 \mathrm{~s}^{[16,17]}$ and $60 \mathrm{~s},{ }^{[18,19]}$ respectively, to advance electron microscopy. While the importance of holography was recognized early on, ptychography has only recently gained broad interest. The term 'ptychography' stems from the Greek word for a fold or crease, akin to the Latin origin of the term convolution. Instead of illuminating a small sample with a featureless plane wave, one uses a small, often highly structured illumination to raster scan an extended sample. The deconvolution of the effects due to the sample from those due to the structured illumination can be ensured if the sample is scanned in sufficiently fine, overlapping steps. ${ }^{[20,21]}$ Yet ptychography became practical only by combining it with iterative phase retrieval algorithms, ${ }^{[22]}$ which reduced the sampling requirements drastically. Since this discovery the field has seen unprecedented research activity: demonstrations of X-ray ptychography, [23] combination with tomography for high-resolution imaging in three dimensions ${ }^{[24]}$ and various method- ological improvements, in particular reducing the dependence on accurate $a$ priori information on illumination or sample positioning, [25-30] have transformed X-ray ptychography into a powerful, robust, and user-friendly technique, for which dedicated instruments are being designed and built at many synchrotrons worldwide.

As in all CDI techniques, ptychographic contrast is inherently quantitative and allows accurate statements about the electron density distribution in the sample, ${ }^{[31]}$ which is trivially related to mass density if the chemical composition is known approximately. In fact, since both $\mathrm{X}$-ray absorption and phase advance are measured, the chemical composition can often be inferred without recourse to spectroscopic scans. ${ }^{[32]}$ X-ray ptychography has resolved high-contrast test structures with $<10 \mathrm{~nm}$ and $<20 \mathrm{~nm}$ resolution in two and three dimensions, respectively. ${ }^{[33-35]}$ Phase sensitivity well below 0.01 radians has been demonstrated ${ }^{[36,37]}$ corresponding to, for instance, sensitivity to layers of less than $100 \mathrm{~nm}$ of light materials such as amorphous carbon or protein, even when encasing ice reduces the contrast. Ptychography finds application in studies of complex composite materials such as cement paste ${ }^{[38]}$ or natural and synthetic fibers ${ }^{[39]}$ and is used in geology, paleontology, catalysis, and increasingly in the life sciences as well.

Fig. 4 illustrates the experimental setup (a) as well as the measurement strategy and outcome (b). The setup requires high instrumental stability and the ability to accurately raster scan the sample with a coherent X-ray beam of up to a few microns in diameter. Without further optics, scattered light is recorded with a $2 \mathrm{D}$ area 
detector. The compound data from multiple known scan positions are inverted to yield an image, whose resolution is limited by the maximum scattering angle at which a signal can still be collected and by the positioning accuracy. High accuracy in measuring on an absolute scale the complex-valued index of refraction in two dimensions or, if combined with tomographic methods, in three dimensions allows highly accurate and often unambiguous segmentation of data, as illustrated in Fig. 5.

Ptychography combines characteristics of both full-field methods described in the first section and scanning microscopy approaches, as described in the following sections. This combination provides flexibility and thus allows to customize imaging strategies to a wide range of samples, including cryogenically protected samples ${ }^{[36,40]}$ and samples whose response to environmental, chemical, or mechanical influences are to be characterized, [39] Ptychographic tomograms with $\sim 10^{8}$ resolution elements have been measured, and acquisition rates

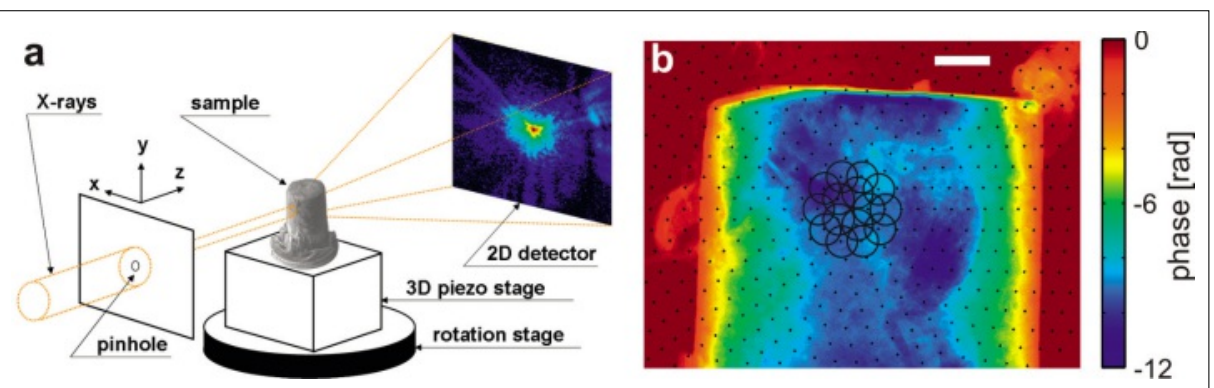

Fig. 4. (a) Sketch of the experimental setup for ptychographic computed tomography (not to scale). A sample is raster scanned with a small coherent X-ray beam, here produced simply by an aperture. The scattered light is imaged without further optics. (b) The reconstructed phase advance of a sample of resin-impregnated, hardened cement paste. The scale bar corresponds to $5 \mu \mathrm{m}$. Circles symbolize the area illuminated at any single scan position; scan positions are symbolized by dots. Acquisition of several such projections as a function of sample orientation allow for tomographic reconstruction of the three-dimensional electron density distribution. Figure adapted from ref. [38].

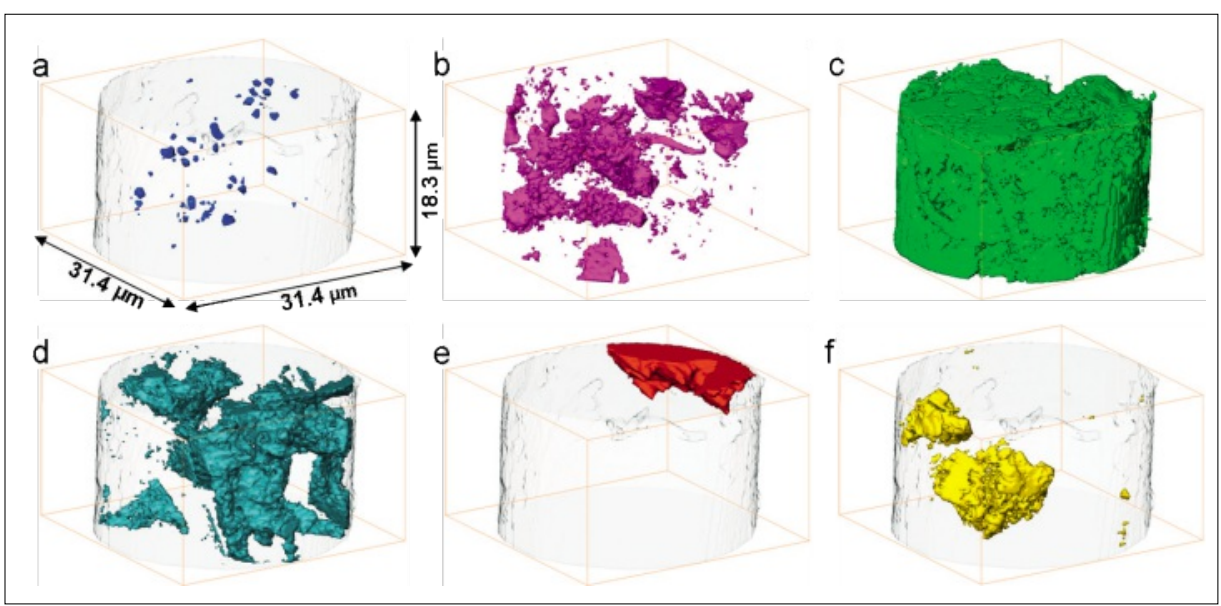

Fig. 5. Identification of various phases in a sample of epoxy resin-impregnated, hardened cement paste. (a) Non-impregnated or partially impregnated porosity, (b) epoxy-impregnated porosity, (c) hydrates, predominantly calcium-silicate-hydrates, (d) calcium hydroxide, (e) calcium carbonate, and $(f)$ unhydrated or partially hydrated clinker residues. Figure adapted with permission from ref. [38]. Copyright Elsevier 2013.
Essentially all material in the X-ray beam path, such as slits, windows, air or residual gas, and the sample container, tend to scatter in the same angular range as the sample itself and obscure the signal of interest. Further, for bio-molecules in solution, the scattering signal of the molecules of interest is weak in comparison to most other signals and drops with the fourth power of the momentum transfer, q.

To position SAXS/WAXS, we briefly sketch similarities and differences between these and other X-ray techniques. WAXS and powder diffraction are basically the same technique apart from the fact that WAXS may be applied to any kind of sample, including amorphous and anisotropic ones. SAXS on macromolecules in solution may be seen as an extension of singlecrystal macromolecular structure determination (MX). It is used to determine the molecular envelope of the molecules in solution and to study conformational changes as a function of system parameters. In terms of the general experimental setup, SAXS is very similar to ptychography in forward scattering geometry, as described in the previous section. The differences are in the sample positioning system and in the utilization of the full available X-ray flux for SAXS, rather than just a small coherent portion of it. Hence SAXS requires a beamstop in front of the detector in order to protect it from the intense primary beam. If SAXS is performed in scanning mode, it is conceptually identical to scanning transmission X-ray microscopy (STXM), as described in the next section. The socalled dark-field signal in STXM performed with a segmented or area detector is a SAXS signal.. ${ }^{[1]}$ Nevertheless, STXM and scanning SAXS instruments look quite different; STXM often uses beams of a few $10 \mathrm{~nm}$ 's in diameter to study correspondingly small sample areas in the soft X-ray range whereas scanning SAXS is often performed at harder X-ray energies with beam diameters of a few to a few hundred micrometers to access correspondingly larger sample areas. The data analysis of the dark field or SAXS signal in STXM is often performed summarily because a detailed analysis is difficult with the statistics of typical data and thus outside the scope of such experiments.

The quantitative analysis of SAXS data has other inherent difficulties. If the illuminated volume encompasses scattering entities with random orientations, then the scattering signal is a spherically symmetric ensemble average and is thus one-dimensional (1D). With such data, 3D structures can only be determined if complementary information on the sample exists. Although more complex systems such as a piece of tissue, polymer, or wood often show scattering of reduced symmetry, such data can 
only be analyzed in a quantitative way if a model or some other prior knowledge on the sample is available. While this may be a weakness, it is a strength of SAXS that it can be applied to basically any kind of ordered or disordered sample, and that often qualitative average information at the nanoscale can be deduced which is not easily accessible otherwise.

For heterogeneous samples, measuring at several discrete sample positions suggests itself. The incident beam can be focused to improve the spatial resolution of the inhomogeneity in the sample. Improving the statistics of spatially resolved measurements at many positions of a sample eventually evolves into imaging: SAXS and WAXS applied in scanning mode are 2D imaging techniques; ${ }^{[42,43]}$ if combined with sample rotation, they become 3D techniques. ${ }^{[44,45]}$ Given the flux of a synchrotron radiation source and recently developed fast X-ray detectors with no readout noise and high dynamic range, millions of SAXS patterns can be recorded in less than 12 hours. With such data volumes, statistical approaches for extracting information from scanning SAXS/WAXS data become attractive. Semi-quantitative information, such as the degree of asymmetry of the scattered intensity as a function of the azimuthal angle, can be determined during data acquisition and provides valuable feedback on the data quality and for the further acquisition strategy. A way of analyzing the data is to treat all SAXS intensity patterns recorded from a sample as a statistical set. From such a set, basic scattering-intensity components can be extracted. This may facilitate the analysis of data for samples composed of multiple materials and modifications of these. The SAXS data can be complemented with information from WAXS experiments to improve the uniqueness of such an analysis. If a model to describe the scattering of the sample is available, then the extraction of a few values of model parameters for each data point will lead to a map, i.e. an image of these parameters across the sample. If the SAXS or WAXS data contain easily separable information, such as diffraction peaks, then this information lends itself to further analysis the result of which may again be represented as maps of the extracted parameters.

A 2D scanning SAXS experiment on polypropylene (PP) and polyvinylidenefluoride (PVDF) is illustrated in Fig. 6. The material was injection-molded. The flow in this process induces orientation of polymer assemblies at different length scales; depending on the material properties and the cooling rates in the different regions of the mold part of this orientation is conserved. In Fig. 6 the orientation of the scattering signal for features in the 8 to $18 \mathrm{~nm}$ range is depicted. While the thin cantilevers extending to the right of each sample exhibit quite uniform orientation at this length scale, a more complex pattern of orientations is observed in the thick support to the left of each sample. This study allowed the uniformity of the cantilevers to be estimated at the nanometer scale and served as one of the tests for determining their suitability as sensors. ${ }^{[46]}$

In conclusion, scanning SAXS accesses the density and orientation of nanoscale features, ensemble-averaged over the illuminated volume and spatially resolved over square-millimeter and centimeter areas and corresponding volumes. It bridges the gap between information retrieved from high-resolution local techniques and information from low-resolution, large field-of-view imaging techniques. SAXS is ideally suited for studies of hierarchically ordered materials in life and materials sciences.

\section{Scanning Transmission X-ray Microscopy}

Scanning transmission X-ray microscopy (STXM) is a relatively simple real- space imaging technique that utilizes a Fresnel zone plate to focus a monochromatic X-ray beam onto a single pixel-sized area of a thin sample and measures the intensity of the transmitted beam in a rasterscanning mode thereby filling an image array. Since STXM requires no objective lens, there is no attenuation of the beam between the sample and the detector and the dose delivered to the sample by this technique is reduced compared to a fullfield, lens-based approach. Furthermore, positioning the optical elements to account for the achromaticity of zone plates, i.e. the dependence of their focal length on the wavelength of light, becomes very simple. The procedure allows imaging with routine spectroscopic techniques at strategically chosen X-ray photon energies. STXM images can cover a wide range of length scales with scan ranges extending to many millimeters. Its maximum resolution currently approaches $10 \mathrm{~nm}$ and is determined largely by the quality of the focusing zone plate.[47]

The strongest advantage of STXM is its ability to utilize near-edge X-ray absorption fine structure (NEXAFS, alternatively XANES) to achieve strong, natural contrast that provides insight into a sample mate-
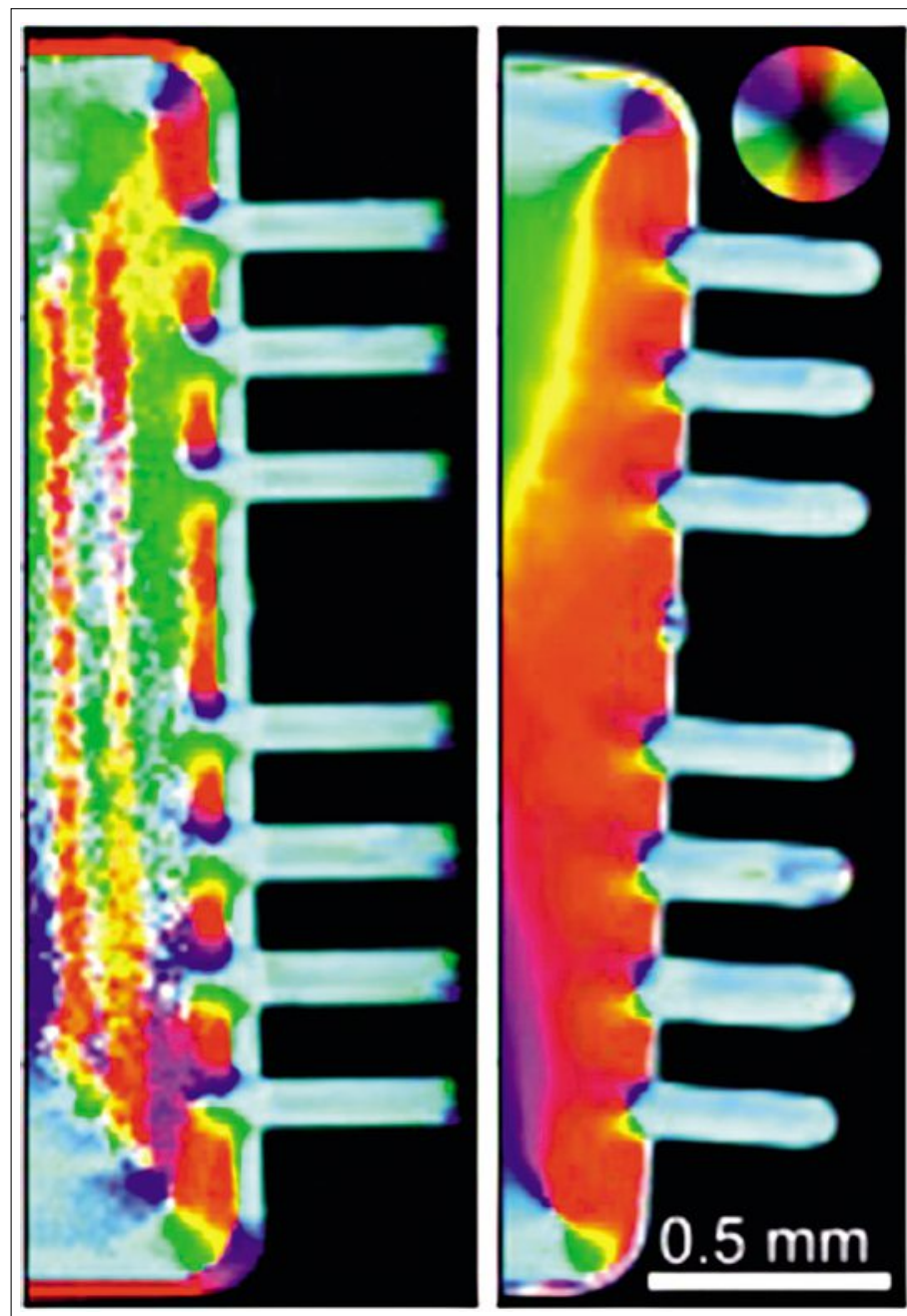

Fig. 6. Orientation of polymer assemblies in injection-molded polymers: Left polypropylene, right polyvinylidenefluoride. Both samples consist of a thick support and approximately $35 \mu \mathrm{m}$ thin cantilevers. The orientation derived from small-angle $X$-ray scattering at each position within the sample area is color-coded according to the disc in the top right. The selected momentum transfer corresponds to $8-18 \mathrm{~nm}$ feature size in direct space. Data from ref. [46]. 
rial. ${ }^{[48,49]}$ Whereas SAXS and WAXS allow the extraction of detailed structural information from the scattering of a relatively large beam, STXM can access very finescale chemical and structural information through spectroscopy. STXM information reflects various details of the electronic structure and the types of photo-excitations that produce resonance signals at energies in the neighborhood of elemental absorption edges.

The near-edge resonances at the carbon K-edge with photon energies (wavelength) of approximately $290 \mathrm{eV}(4.3 \mathrm{~nm})$, provide excellent contrast between different organic materials. These spectral features are due to photo-induced transitions from the carbon 1s state into unoccupied electronic states associated with the antibonding molecular orbitals and thus provide information on the chemical bonds that the carbon atoms are participating in. In other words, by utilizing spectroscopy at the carbon K-edge, STXM achieves image contrast based on molecular structure - the defining attribute of organic materials. The method is quantitative and does not require labeling. Fig. 7 demonstrates this principle with images of a thin film composed of a blend of poly(9,9-dioctylfluorene-cobis(N,N-(4-butylphenyl))bis(N,N-phenyl-1,4-phenylene)diamine) (PFB) and poly(9,9-dioctylfluorene-co-benzothiadiazole) (F8BT). The raw transmission images (black and white images in the lower left) were measured at photon energies corresponding to the pre-edge $(280 \mathrm{eV})$, resonant (F8BT:284.5 eV, PFB:285 eV) and post-edge $(320 \mathrm{eV})$ spectral features as indicated in the spectra by the vertical green lines. These variations in contrast are especially noticeable in the $285 \mathrm{eV}$ image that corresponds to the sharp, intense $\pi^{*}$ resonance of PFB. From the knowledge of the spectra of the components, a quantitative composition map (Fig. 7, right) as well as a map of the sample thickness can easily be calculated.

STXM has also been used to map the orientation of molecules within a given sample area by utilizing linear dichroism, a spectroscopic effect in which the intensity of a resonance depends on the orientation of the transition dipole moment relative to the electric field vector of the linearly polarized X-ray beam. Successive images measured at the photon energy of a dichroic resonance for different azimuthal orientations of the sample with respect to the polarization axis of the X-rays reveal a cosine-squared relationship whose phase and magnitude indicate the preferred molecular orientation and degree of ordering, respectively, in the imaged area. ${ }^{[51-53]}$ The raw transmission images in Fig. 8 (left) demonstrate such dichroic imaging. They have been measured at the F8BT $\pi^{*}$ di-

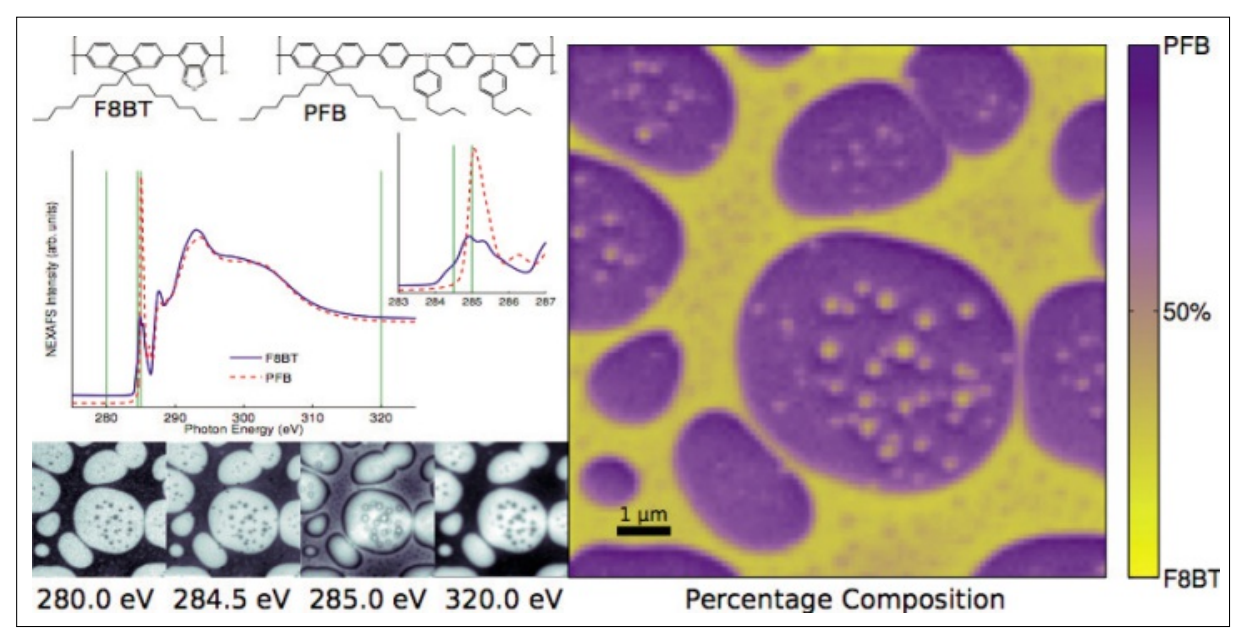

Fig. 7. Molecular structure (upper left) and NEXAFS spectra (centre left) of PFB and F8BT materials. Raw absorption images (lower left) of a PFB:F8BT blend, taken at photon energies of 280, $284.5,285$ and $320 \mathrm{eV}$, show variations in contrast that can be combined with knowledge about the spectra of PFB and F8BT to produce a quantitative composition map (right). Adapted from ref. [50].

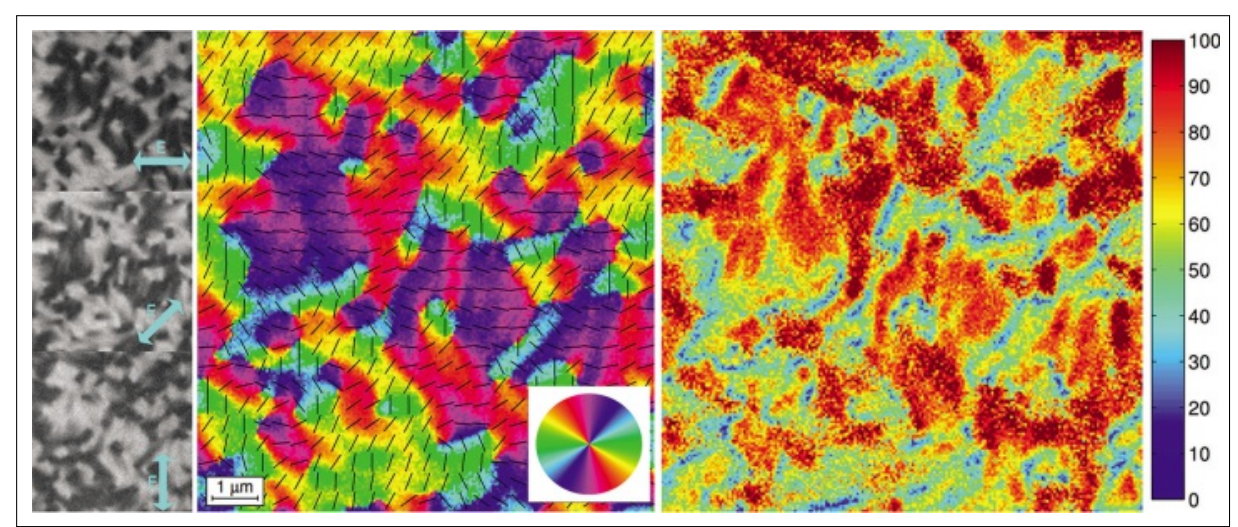

Fig. 8. Three raw transmission images (left) taken with different orientations of the linear polarization axis of the probing X-ray beam show varying contrast. The images can be combined to provide maps of the NEXAFS resonance orientation (center, polymer chain orientations indicated by lines) and the degree of molecular alignment (right). Adapted from ref. [53].

chroic resonance, with the electric field vector of the $\mathrm{X}$-ray beam at relative angles of $0^{\circ}, 45^{\circ}$, and $90^{\circ}$. Note the inversion of contrast between the $0^{\circ}$ and $90^{\circ}$ images. The color map in the center of Fig. 8 presents a map of the orientation of the F8BT $\pi^{*}$ resonance, from which the orientation of the polymer chains can be inferred, as indicated by the added black line segments. The map on the right of Fig. 8 quantitatively illustrates the degree of molecular alignment. ${ }^{[53]}$

Contrast in STXM can be achieved through several mechanisms, e.g. based on oxidation state ${ }^{[54,55]}$ or fluorescence from trace elements. ${ }^{[56,57]}$ Electron detection has been used to map surface composition. ${ }^{[50,58]}$ Furthermore, magnetic domains can be imaged based on X-ray magnetic circular dichroism and their dynamics followed in pump-probe experiments. ${ }^{[48,59]}$ Phase contrast is also possible, although absorption contrast is often more advantageous in the soft X-ray region of the spectrum where STXM experiments are commonly con- ducted. ${ }^{[41,60]}$ With such diverse contrast mechanisms, STXM lends itself well to a wide range of investigations in almost every scientific discipline.

\section{Conclusion and Outlook}

X-ray imaging at synchrotron facilities provides a wealth of techniques for studying structures of a wide range of material classes in the nano- to micrometer range. The high flux of synchrotron X-ray beams combined with high-speed detectors enable dynamic imaging at time scales from nanoseconds, as is typical for magnetization dynamics, to seconds and minutes, as is characteristic of wet foam expansions. Owing to the high penetration depth of $\mathrm{X}$-ray beams it is also possible to perform $\mathrm{X}$-ray experiments with in situ environmental cells in order to study materials under specific temperature, pressure, and chemical conditions including exposure to gasses or liquids. 
Samples that are either static or changing on time-scales down to the millisecond range, are best imaged with a continuous $\mathrm{X}$-ray source. For better time resolution, as is typically needed in pump-and-probe type studies, a pulsed source is mandatory; often the bunch structure of the synchrotron radiation sources can be exploited. Free-electron lasers (FELs) open up new possibilities for such experiments because of their highly intense pulses with lengths in the femtosecond range, and repetition rates from a few $\mathrm{Hz}$ up to $\mathrm{kHz}$. As discussed by B. Patterson et al. in this issue, ${ }^{61]}$ the massive improvements in peak brightness and pulse duration of FEL radiation will enable new types of experiments ranging from the study of the structure and lifetime of short-lived molecular states in solution or on a catalytic surface to the investigation at atomic resolution of the structure of biological and inorganic nanostructures, to mention but a few. While the X-ray freeelectron lasers readily take the crown in some measures of performance, the large and steady throughput of synchrotron facilities will continue to serve the bulk of the X-ray community. Thus, the two radiation sources, rather than being competitors, complement each other. The future of X-ray imaging at synchrotron sources looks bright.

Received: November 28, 2013

[1] K. S. Mader, P. Schneider, R. Muller, M. Stampanoni, Bone 2013, 57, 142.

[2] K. Mader, R. Mokso, C. Raufaste, B. Dollet, S. Santucci, J. Lambert, M. Stampanoni, Colloid Surface A 2012, 415, 230

[3] C. David, B. Nohammer, H. H. Solak, E. Ziegler, Appl. Phys. Lett. 2002, 81, 3287.

[4] F. Pfeiffer, T. Weitkamp, O. Bunk, C. David, Nat. Phys. 2006, 2, 258.

[5] T. Weitkamp, A. Diaz, C. David, F. Pfeiffer, M. Stampanoni, P. Cloetens, E. Ziegler, Opt. Express 2005, 13, 6296.

[6] S. A. McDonald, F. Marone, C. Hintermuller, G. Mikuljan, C. David, F. Pfeiffer, M. Stampanoni, J. Synchr. Rad. 2009, 16, 562.

[7] F. Pfeiffer, M. Bech, O. Bunk, P. Kraft, E. F. Eikenberry, C. Bronnimann, C. Grunzweig, C. David, Nat. Mater. 2008, 7, 134.

[8] P. Modregger, M. Stampanoni, in 'Emerging imaging technologies in medicine', Eds. M. A. Anastasio, P. J. La Riviere, Taylor and Francis Book, Inc, Chicago, 2012.

[9] A. Sakdinawat, D. Attwood, Nat. Photonics 2010, 4,840

[10] S. R. Wu, Y. Hwu, G. Margaritondo, Materials 2012, 5, 1752.

[11] H. N. Chapman, K. A. Nugent, Nat. Photonics 2010, 4,833 .
[12] J. F. van der Veen, F. Pfeiffer, J Phys-Condens. Mat. 2004, 16, 5003.

[13] H. N. Chapman, A. Barty, M. J. Bogan, S. Boutet, M. Frank, S. P. Hau-Riege, S. Marchesini, B. W. Woods, S. Bajt, W. H. Benner, Nat. Phys. 2006, 2,839 .

[14] S. Eisebitt, J. Luning, W. F. Schlotter, M. Lorgen, O. Hellwig, W. Eberhardt, J. Stohr, Nature 2004, 432, 885.

[15] G. J. Williams, H. M. Quiney, B. B. Dhal, C. Q. Tran, K. A. Nugent, A. G. Peele, D. Paterson, M. D. de Jonge, Phys. Rev. Lett. 2006, 97, 025506.

[16] D. Gabor, Nature 1948, 161, 777.

[17] D. Gabor, Proc. Roy. Soc. Lond. A 1949, 197, 454.

[18] R. Hegerl, W. Hoppe, Berich. Bunsen Gesell. 1970, 74, 1148

[19] W. Hoppe, Acta Cryst. A 1969, 25, 508.

[20] H. N. Chapman, Ultramicroscopy 1996, 66, 153.

[21] J. M. Rodenburg, R. H. T. Bates, Philos. Trans. Roy. Soc. A 1992, 339, 521.

[22] J. M. Rodenburg, A. C. Hurst, A. G. Cullis, Ultramicroscopy 2007, 107, 227.

[23] J. M. Rodenburg, A. C. Hurst, A. G. Cullis, B. R. Dobson, F. Pfeiffer, O. Bunk, C. David, K. Jefimovs, I. Johnson, Phys. Rev. Lett. 2007, 98 , 034801.

[24] M. Dierolf, A. Menzel, P. Thibault, P. Schneider, C. M. Kewish, R. Wepf, O. Bunk, F. Pfeiffer, Nature 2010, 467, 436.

[25] M. Guizar-Sicairosm, J. R. Fienup, Opt. Express 2008, 16, 7264 .

[26] A. M. Maiden, M. J. Humphry, M. C. Sarahan, B. Kraus, J. M. Rodenburg, Ultramicroscopy 2012, 120, 64 .

[27] A. M. Maiden, J. M. Rodenburg, Ultramicroscopy 2009, 109, 1256.

[28] P. Thibault, M. Dierolf, A. Menzel, O. Bunk, C. David, F. Pfeiffer, Science 2008, 321, 379.

[29] P. Thibault, A. Menzel, Nature 2013, 494, 68.

[30] F. Zhang, I. Peterson, J. Vila-Comamala, A. Diaz, F. Berenguer, R. Bean, B. Chen, A. Menzel, I. K. Robinson, J. M. Rodenburg, Opt. Express 2013, 21, 13592.

[31] A. Diaz, P. Trtik, M. Guizar-Sicairos, A. Menzel, P. Thibault, O. Bunk, Phys. Rev. B 2012, 85, 020104(R).

[32] H. Yan, Y. S. Chu, J. Maser, E. Nazaretski, J. Kim, H. C. Kang, J. J. Lombardo, W. K. S. Chiu, Sci. Rep. 2013, 3, 1307.

[33] A. Schropp, R. Hoppe, J. Patommel, D. Samberg, F. Seiboth, S. Stephan, G. Wellenreuther, G. Falkenberg, and C. G. Schroer, Appl. Phys. Lett. 2012, 100, 253112.

[34] J. Vila-Comamala, A. Diaz, M. Guizar-Sicairos, A. Mantion, C. M. Kewish, A. Menzel, O. Bunk, C. David, Opt. Express 2011, 19, 21333.

[35] M. Holler, Sci. Rep. 2014, 4, 3857.

[36] E. Lima, A. Diaz, M. Guizar-Sicairos, S. Gorelick, P. Pernot, T. Schleier, A. Menzel, J. Microscopy 2013, 249, 1 .

[37] Y. Takahashi, A. Suzuki, S. Furutaku, K. Yamauchi, Y. Kohmura, T. Ishikawa, Appl. Phys. Lett. 2013, 102, 094102.

[38] P. Trtik, A. Diaz, M. Guizar-Sicairos, A. Menzel, O. Bunk, Cement Concrete Comp. 2013, 36, 71.

[39] M. Esmaeili, J. B. Fløystad, A. Diaz, K. Høydalsvik, M. Guizar-Sicairos, J. W. Andreasen, D. W. Breiby, Macromol. 2013, 46, 434.
[40] R. N. Wilke, M. Priebe, M. Bartels, K. Giewekemeyer, A. Diaz, P. Karvinen, T. Salditt, Opt. Express 2012, 20, 19232.

[41] A. Menzel, C. M. Kewish, P. Kraft, B. Henrich, K. Jefimovs, J. Vila-Comamala, C. David, M. Dierolf, P. Thibault, F. Pfeiffer, O. Bunk, Ultramicroscopy 2010, 110, 1143.

[42] O. Bunk, M. Bech, T. H. Jensen, R. Feidenhans'l, T. Binderup, A. Menzel, F. Pfeiffer, New J. Phys. 2009, 11, 123016.

[43] P. Fratzl, H. F. Jakob, S. Rinnerthaler, P. Roschger, K. Klaushofer, J. Appl. Crystallogr. 1997, 30, 765 .

[44] T. H. Jensen, M. Bech, O. Bunk, A. Menzel, A. Bouchet, G. L. Duc, R. Feidenhans'1, F. Pfeiffer, Neuroimage 2011, 57, 124.

[45] Y. F. Liu, I. Manjubala, P. Roschger, H. Schell, G. N. Duda, P. Fratzl, Xiv International Conference on Small-Angle Scattering (Sas09) 2010, 247.

[46] P. Urwyler, H. Deyhle, O. Bunk, P. M. Kristiansen, B. Müller, J. Appl. Phys. 2012, 111, 103530.

[47] J. Vila-Comamala, K. Jefimovs, J. Raabe, T. Pilvi, R. H. Fink, M. Senoner, A. Maassdorf, M. Ritala, C. David, Ultramicroscopy 2009, 109, 1360.

[48] H. Ade, H. Stoll, Nat. Mater. 2009, 8, 281.

[49] B. Watts, H. Ade, Mater. Today 2012, 15, 148.

[50] B. Watts, C. R. McNeill, Macromol. Rapid Comm. 2010, 31, 1706

[51] S. Kowarik, K. Broch, A. Hinderhofer, A. Schwartzberg, J. O. Osso, D. Kilcoyne, F. Schreiber, S. R. Leone, J. Phys. Chem. C 2010, 114, 13061.

[52] T. Schuettfort, B. Watts, L. Thomsen, M. Lee, H. Sirringhaus, C. R. McNeill, ACS Nano 2012 , $6,1849$.

[53] B. Watts, T. Schuettfort, C. R. McNeill, $A d v$. Funct. Mater. 2011, 21, 1122.

[54] J. Miot, K. Benzerara, G. Morin, A. Kappler, S. Bernard, M. Obst, C. Ferard, F. Skouri-Panet, J. M. Guigner, N. Posth, M. Galvez, G. E. Brown, F. Guyot, Geochim. Cosmochim. Acta 2009, 73, 696.

[55] S. Takahama, S. Gilardoni, L. M. Russell, J. Geophys. Res.-Atmos. 2008, 113, D14205.

[56] A. P. Hitchcock, M. Obst, J. Wang, Y. S. Lu, T. Tyliszczak, Environ. Sci. Technol. 2012, 46, 2821.

[57] B. Kaulich, A. Gianoncelli, A. Beran, D. Eichert, I. Kreft, P. Pongrac, M. Regvar, K. Vogel-Mikus, M. Kiskinova, J. R. Soc. Interface 2009, 6, S641.

[58] C. Hub, S. Wenzel, J. Raabe, H. Ade, R. H. Fink, Rev. Sci. Instru. 2010, 81, 033704.

[59] A. Fernandez-Pacheco, L. E. Serrano-Ramon, T. Tyliszczak, K. W. Chou, R. Cordoba, A. Szkudlarek, L. O. Brien, C. Kapusta, M. R. Ibarra, J. M. De Teresa, Nanotechnol. 2012, 23, 105703.

[60] J. Raabe, B. Watts, G. Tzvetkov, R. H. Fink, C. Quitmann, J. Phys. Conf. Ser. 2009, 186, 012012.

[61] B. D. Patterson, P. Beaud, H. H. Braun, C. Dejoie, G. Ingold, C. Milne, L. Patthey, B. Pedrini, J. Szlachentko, R. Abela, Chimia 2014, $68,73$. 